\title{
e-Phaïstos
}

e-Phaïstos

Revue d'histoire des techniques / Journal of the history

of technology

IV-1 | 2015

Les arts de guerre et de grâce (XIVe-XVIIle siècles)

\section{Entre mouvements intuitifs et gestes techniques : « Poignarder » un homme $\left(\mathrm{XV}^{\mathrm{e}}-\mathrm{XVI}^{\mathrm{e}}\right.$ siècles $)$}

Between intuitive movements and technical gestures: "stabbing" a man (from the 15th to the 16th century)

\section{Pierre-Henry Bas}

\section{(2) OpenEdition}

\section{Journals}

\section{Édition électronique}

URL : http://journals.openedition.org/ephaistos/633

DOI : 10.4000/ephaistos.633

ISSN : 2552-0741

\section{Éditeur}

IHMC - Institut d'histoire moderne et contemporaine (UMR 8066)

\section{Édition imprimée}

Date de publication : 1 avril 2015

Pagination : 43-55

ISSN : 2262-7340

\section{Référence électronique}

Pierre-Henry Bas, «Entre mouvements intuitifs et gestes techniques : «Poignarder » un homme (xveXVI Ie siècles) », e-Phaïstos [En ligne], IV-1 | 2015, mis en ligne le 22 novembre 2016, consulté le 30 avril 2019. URL : http://journals.openedition.org/ephaistos/633 ; DOI : 10.4000/ephaistos.633 


\title{
Entre mouvements intuitifs et gestes techniques: "Poignarder" un homme ( $\mathbf{X V}^{\mathrm{e}}-\mathrm{XVI}^{\mathrm{e}}$ siècles)
}

\author{
Pierre-Henry Bas \\ Institut de Recherches Historiques du \\ Septentrion \\ Université de Lille 3
}

Les dagues, couteaux et autres poignards semblent appartenir à une catégorie en tous points opposée à l'épée. Si cette dernière possède un caractère empreint de noblesse et de justice ${ }^{1}$, la symbolique du poignard est toute autre : c'est celle de la colère et du meurtre ${ }^{2}$. Cette image se retrouve dans les lettres de rémission de la fin du Moyen Âge et de la Renaissance. Ces lettres patentes données par une autorité afin d'absoudre l'auteur d'un crime appelé le suppliant- avant son jugement montrent que les dagues et les couteaux sont des objets communs portés par toutes les catégories sociales, le plus souvent pour des raisons d'utilité quotidienne (c'est le cas du petit couteau taille pain). Certains ont l'allure d'atour symbolique, à l'exemple des dagues portées entre les jambes 3 aux formes phalliques parfois assez évidentes 4 . Mais les dagues et les couteaux sont également des armes de fortune en cas de rixe ou d'agression, c'est pourquoi les textes normatifs en interdisent le port, ou n'autorisent seulement que des lames d'une certaine longueur ou démunies d'une pointe trop aiguë5. Ces interdictions semblent rarement respectées et l'ensemble de la population est susceptible de se servir de ce type d'armes dès que le danger guette $^{6}$. Cette présence quotidienne des dagues conduit à leurs fréquentes utilisations en cas de conflit. Ceci explique sans doute aussi l'enseignement du combat avec cet équipement par les maîtres d'armes, que l'on retrouve dans les livres d'armes ou livres de combat germaniques (Fechtbücher) datant de la même période7.

Ainsi, cette double représentation, dans des sources pourtant si différentes conduit à nous interroger sur son utilisation technique et à se demander si l'emploi martial d'une dague ou d'un couteau est universel, ou bien en quoi il peut être influencé par la spécificité du contexte, lorsque celui-ci est aussi sérieux qu'une rixe, ou bien au contraire ludique. Il s'agit alors d'étudier les différentes armes utilisées, de comprendre quels sont les gestes techniques associés et enfin de montrer comment la pratique expérimentale permet de saisir l'importance de certains facteurs propres à des situations particulières.

\section{Les armes utilisées}

Pour les hoplologues - les spécialistes des armes - contemporains, il est parfois assez difficile de faire la distinction entre un couteau, une dague ou un poignard. D'un point de vue fonctionnel, le couteau serait utilisé pour couper ou entailler, tandis que la dague et le poignard serviraient à piquer ou à estoquer. Or ces fonctions restent générales. Si l'on se réfère aux textes normatifs, il semblerait que l'on distingue à l'époque d'une part les lames symétriques des dagues qui ont la forme de petites épées et, d'autre part, les manches plats des couteaux, montés à plate semelle, c'est-à-dire avec un manche formé par deux plaques munies de clous de fixa- 
tion : Couteau à manche plat dont les lames sont faites en forme de dague ou de poignard ${ }^{8}$. À l'opposé, certaines lames de couteau sont montées avec une soie enfoncée dans un manche, comme on le ferait pour les épées. Certains spécialistes ont choisi le terme anglais de knife-daggers pour désigner les armes appartenant aux deux catégories9. En ce qui concerne les lettres de rémission, les auteurs ne font pas nécessairement la distinction entre les deux instruments et peuvent alterner les deux termes pour la même arme ${ }^{10}$. La distinction semble davantage résider dans l'action : l'outil sert à couper ou à piquer sa nourriture, l'arme sert uniquement à blesser un individu. Hormis la longueur de la lame qui a son importance au combat, on note plusieurs éléments montrant une orientation à des fins martiales. Tout d'abord les éléments servant à optimiser le coup, comme un pommeau pour l'équilibre ou un affinement de la lame pour une meilleure pénétration, à l'exemple des dagues très effilées que nous appelons aujourd'hui miséricordes $^{11}$. En plus d'empêcher la main de glisser lors d'une perforation, les deux quillons qui forment la garde servent aussi se protéger des coups adverses. Il en va de même pour les rouelles, ces rondelles métalliques formant la garde ou le pommeau. $\mathrm{Au}$ $\mathrm{XV}^{\mathrm{e}}$ siècle on trouve parfois à côté du terme couteaux à pointes ${ }^{12}$, celui de dollequin ${ }^{13}$ et aussi de poignart $^{14}$. Ce dernier va s'imposer au XVI ${ }^{e}$ siècle dans les livres de combat ${ }^{15}$. On le considère à l'origine comme une «dague de combat $»^{16}$. Par la suite, certains auteurs comme Paul-Hector Mair ${ }^{17}$ ne parlent plus de dague, mais seulement du poignard: dolchen ${ }^{18}$, un nom bien proche du dollequin...19

\section{Leur utilité dans les affaires sérieuses}

En plus de faciliter son transport, la taille réduite de ce type d'arme oblige à avoir une distance effective en combat (la mesure) très proche de l'ennemi, ce qui permet des gestes relativement précis et rapides, exécutables par exemple dans le cas d'un assassinat ou d'une tentative de meurtre :

« [...] Une matinée, par voye de fait et d'aguet apensé, vint audit feu [Bouchart père] qui lors estoit de l'aage de LXXVI ans et plus, et par derriere, ainsi qu'il yssoit de sa maison, lui donna sept coups de dague, et telement le navra que cinq jours après il ala de vie a trespas. ${ }^{20}$

Les dagues et les couteaux sont utilisables en dernier recours, lorsque l'adversaire est trop proche et que l'on ne peut plus utiliser efficacement aucune arme d'hast, ou d'armes à une main, comme les épées et les longs couteaux ${ }^{21}$ :

« [...] [Wattier Nolet] darda d'une javeline apres duquel cop il ne lui fit aucun mal et tira incontinent ung grant couteau qu'il avoit, duquel il navra ledit Micquiel [de Le Mote] en la cuisse et se prindrent l'un a l'autre tellement que ledit suppliant se deffeist de son dit cousteau, mais d'une petite daggue qu'il avoit en sa chainture, il bailla deux cops audit Micquiel l'un environ la mamelle et l'autre entour la chainture. Des quelles navrures ledit Micquiel termina tost apres vie par mort. $»^{22}$

Ces armes sont également dégainées pour la lutte au sol, principalement celui qui advient lors des pas d'armes et les duels réglementés, et de tous les combats en harnois (harnischfechten) en général. Elles permettent d'estoquer au cou, au visage ou à l'aine, en passant par les défauts de la cuirasse de l'adversaire préalablement immobilisé au sol'23. Certaines lettres de rémission mentionnent cette position où un coup d'estoc permet de mettre fin à ce type de situation plus qu'oppressante :

« [...] « Je vieulx luyter a toy et savoir qui sera le plus fort ". A quoy fut dit par ledit Thomas, suppliant : «Je le te [sic] accorde ». Et sur ses parolles, s'entreprindrent au collet pour luyter [...] Et apres quelzques prinses et secousses qu'ilz donnerent les ungs aux autres, icelluy Maillard, qui estoit robuste, fort et puissant homme, gecta ledit Thomas, suppliant, sur le doz soubz luy. Et en ce faisant, poussa de sa pesanteur et de sa force sur l'estomac dudit Colas Meglat [sic: Thomas] Le Bastard, ayant l'un de ses genoulx sur le bas de son ventre. Sur quoy il se tinst assez longuement et plus beaucoup qui ne luy estoit permys selon l'usaige et maniere de lute. En maniere que ledit Thomas Le Bastard, suppliant, qui se sentoit grevé de ladite cheuste et oultrage que en ce faisant luy fist ledit 
Maillart, se despita et prinst ledit Maillart aux cheveulx pour le faire vaster de dessus luy et s'esvertua pour soy oster et lever. Et ledit Maillard par semblable saisit ledit Thomas aux cheveulx avec une main et par la gorge avec l'autre, disant : "Par le Sang-Dieu, villain, tu ne m'es pas trop fort ! » Et sur ce propoux, tenoit de si court ledit Thomas, suppliant, qu'il perfoit puissance et alayne. [...] Icelluy Maillard perseverant en sa fureur plus estroictement prochassa ledit Thomas. Lequel voyant sa force n'estre sienne et que ledit Maillart le tenoit de si court, tira ung cousteau pointu qu'il avoit a sa saincture, duquel il avoit de coustume tailler son pain, pour en frapper ledit Maillart affin de luy faire lascher sa prinse et eviter le danger en quoy il estoit. »24
Cet emploi dans un contexte que l'on pourrait qualifier de défense personnelle est également présent dans les livres de combat, car la dague « a beaucoup d'avantages, alors qu'elle sert à préserver la vie ${ }^{25}$ » afin de se défendre en cas d'agression fortuite. A l'inverse, de nombreuses techniques concernent le combat à mains nues contre un adversaire équipé d'une dague ${ }^{26}$. L'utilisation des différentes clefs de bras, des prises et des projections au sol témoignent du rôle propédeutique de la dague. Son enseignement permet le passage de l'escrime à la lutte en introduisant certaines notions communes aux deux arts ${ }^{27}$. Cependant, le combat à la dague ou

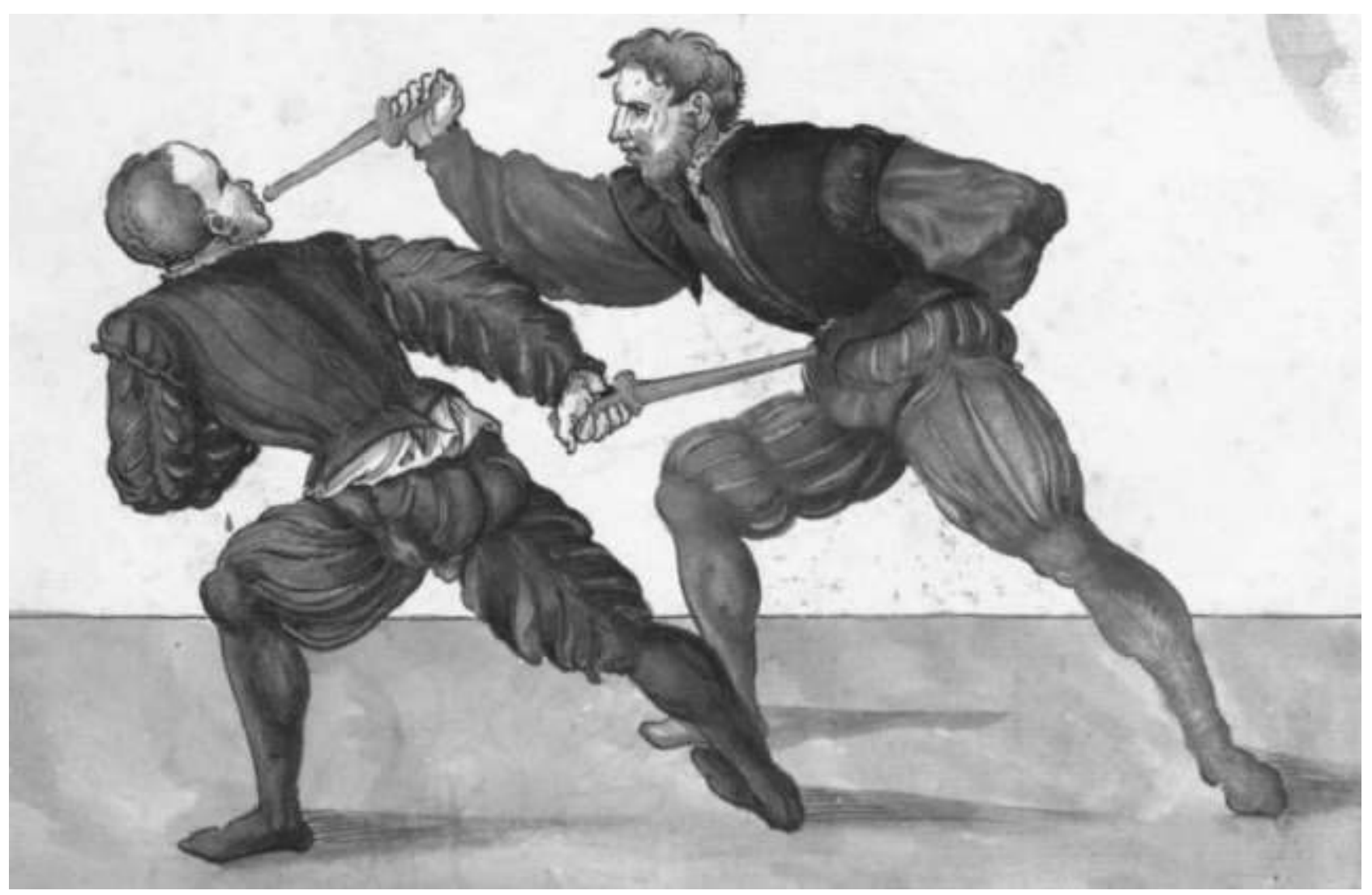

Figure 1 : P.-H. MAIR, milieu du XVIe siècle, Dresde, Sachsische Landesbibliothek, ms. Dresd. C.94, pl.15, $f^{\circ} 1 r^{\circ}$. 
au poignard ne se résume pas aux différents estocs. L'une des tactiques peut être de renverser son adversaire au sol (werfen), de le désarmer (tolchennemen), ou encore de lui briser le bras (armbruch) après une clef articulaire. Mais dans la réalité, c'est à la fois le contexte et le matériel utilisé qui vont déterminer ces différentes possibilités, en fonction de certaines règles et de certaines conventions informelles. On observe dans les ouvrages de PaulHector Mair l'usage d'un simulateur de poignard ayant la forme d'une dague à rouelle en bois, munie d'un embout arrondi utilisé dans le cadre de l'entrainement ou d'une pratique ludique ou protosportive $^{28}$ (Figure 1). L'absence de tranchant n'empêche pas de se servir de la lame afin d'agir sur les articulations de l'adversaire lors des clefs 29 et on peut imaginer l'efficacité de ce genre d'action avec un tranchant aiguisé. Mais cette absence permet aussi d'entreprendre des actions qui semblent risquées avec une vraie arme aiguisée, comme le fait de saisir sa propre lame ou celle de l'adversaire avec la main ${ }^{30}$.

\section{Les gestes offensifs}

Les descriptions dans les livres de combats relatent des affrontements l'arme à la main ; tout au contraire, les lettres de rémissions décrivent le moment où les combattants dégainent leurs dagues, ce qui suppose tout un protocole qui vise probablement à intimider l'adversaire. D'un point de vue technique, la façon dont l'arme est portée et son dégainement déterminent la manière dont elle sera tenue et utilisée, et dans la plupart des cas, la direction des coups et la localisation des blessures. Il y a en effet deux manières de tenir une dague ou un couteau : la première, comme un "pic à glace », la lame près de l'auriculaire afin de donner un coup d'estoc du haut vers le bas (oberstich) ${ }^{31}$ et l'autre, la lame dans le prolongement du pouce pour estoquer $\mathrm{du}$ bas vers le haut (unterstich) ${ }^{32}$, à la manière d'une épée. La position de l'unique tranchant, à l'intérieur ou à l'extérieur, va aussi déterminer l'optimisation de certaines techniques.

Malgré toutes ces considérations sur les coups et leur nature, les lettres de rémission ne sont pas suffisamment précises pour déterminer avec certitude la manière dont l'arme est tenue. Seul les coups assénés avec le plat de la lame, dans l'intention de ne pas blesser trop gravement, sont certainement donnés en tenue "épée »33. Pour les autres, on remarque simplement qu'ils sont davantage portés vers la partie supérieure du corps que vers la partie inférieure, ce qui conduirait à privilégier la tenue en «pic à glace »34. Ce qui détermine l'efficacité d'un coup, c'est-à-dire son potentiel pour entraîner la mort, est la conjoncture de plusieurs paramètres, en particulier celui de sa localisation. Certains coups parviennent par exemple au cœur en passant entre les côtes 35 , alors que d'autres, plus fréquemment, atteignent l'artère sous-clavière ${ }^{36}$. Les coups peuvent également être mortels à cause de leurs conséquences post-traumatiques comme dans le cas d'une infection ou une hémorragie. C'est souvent le cas de ceux touchant l'artère fémorale sans qu'il n'y ait eu, a priori, volonté de tuer ${ }^{37}$. Outre la localisation, la dangerosité d'un coup peut dépendre aussi de la profondeur de la blessure infligée et donc de la force qui lui a été allouée.

Or, ces différents paramètres ne sont jamais exprimés dans les livres de combat, lesquels expliquent peut-être avant tout l'art de l'escrime, non pas celui de tuer, a moins que ce savoir ne soit implicite. Il est donc difficile de distinguer exactement les attaques qui viseraient à blesser mortellement son adversaire et celles qui chercheraient simplement à l'atteindre. Ainsi, si dans l'introduction du combat à la dague, Paul-Hector Mair précise que l'on doit frapper avec tout le bras et pas seulement avec l'avant-bras ${ }^{38}$, c'est sans doute aussi, comme le montrent nos expériences pratiques 39 , pour augmenter sa force et amenuiser la défense adverse. Il ne s'agit pas indubitablement d'atteindre l'adversaire plus durement. Il en va de même quand on fait la distinction entre les coups puissants qui 
vont être portés avec tout le corps, en marchant avec une jambe et en accompagnant avec les hanches, et les coups plus rapides portés uniquement avec le haut du corps ou le bras, en " pompant ${ }^{40}$. Il y a alors comme aujourd'hui une grande différence entre la théorie et la pratique. Le cadre ludique du XVI ${ }^{\mathrm{e}}$ siècle, avec le développement des Fechtschulen, ces " écoles de combat 》 ou compétitions d'escrime ${ }^{41}$, semble encourager la multiplicité des techniques, en présentant des problèmes originaux propres à une activité proto-sportive ou purement théorique. À l'opposé, les auteurs du XVe siècle $^{42}$ proposent dans leur démarche de codification un système principalement basé sur quelques estocs fondamentaux et les moyens de s'en défendre. En effet, l'art du combat à la dague se comprend dans son aspect défensif. Il s'agit alors d'assimiler les gestes offensifs afin avant tout de savoir les contrecarrer43. Hormis quelques «estocs libres » dans les planches de Hans Talhoffer 44 , les actions des deux protagonistes semblent des plus pertinentes, mais toujours un peu trop conventionnelles : l'attaque est franche et les feintes sont rares.

\section{Les gestes défensifs}

Certaines actions sont communes aux deux types de sources, comme le fait de tenir son adversaire par le col avec la main gauche afin de le frapper ou les solutions pour l'en empêcher45. Mais à la différence de la littérature technique, les lettres témoignent du fait que les improvisations sont prépondérantes. Le désarmement, par exemple, n'est pas toujours un choix tactique :

«[...] [Julian de Mafle] tira sa dague sur ledit suppliant [Colart Dault] lequel n'avoit aucune chose pour soy deffendre que ses mains wuydes et fut contraint pour sa deffense de se dreschier allencontre ledit Julian [de Mafle] et mectre ses bras et mains audevant le cop et lui empoingna le poing oquel il avoit ladicte dague et la lui osta et print. »46

Dans ces lettres les individus ne cherchent pas à renverser leur adversaire ou à lui faire une clef articulaire. Les coups peuvent se donner simultané- ment et sans prendre en compte l'action de l'adversaire, au risque de se blesser mutuellement ou individuellement :

«[...] Ledit [Guilet] Vire cuida ferir de son dit baston ledit suppliant [Guillot Preau], pour laquelle chose reppeller et en soi deffendant, lequel suppliant fery un autre cop de sondiz coustel ledit Vire par le costé, duquel cop après et qu'il eust este confesse et fait son ordonnance comme l'on dit, il

ala assez tost après de vie a trespassement. » 47

Ces gestes sont soit des attaques données pendant celle de l'adversaire (coup de temps), soit simplement des gestes réflexes de protection avec l'arme comme le serait une parade :

« [...] Ledit Vasquin Le Son chauldement print et amassa une pierre. Et icelle tenant en sa main, vint contre ledit suppliant, haulsant le bras, ramenant son coup avec ladite pierre contre ledit suppliant. Lequel suppliant mist au-devant du coup ung cousteau a trancher pain qu'il tenoit en sa main. Et advint que ledit Vasquin, en ramenant sondit coup, eut dudit cousteau que ledit suppliant mist audevant, le bras persé au-dessus du coude. „48

La perte de quelques doigts ou une main percée après une tentative avortée de désarmement ou d'interception, montrent aussi les difficultés pour arrêter efficacement un coup 49 .

\section{Expérimentation gestuelle, du livre de combat à l'expérimentation}

L'expérimentation gestuelle ou le fait d'analyser un geste par le biais de sa pratique et de sa mise en situation, avec des simulateurs adaptés, permet de comprendre que certains paramètres ne semblent pas être abordés dans les livres de combat, alors qu'ils peuvent s'exprimer en partie dans les lettres de rémission. Prenons l'exemple de l'interception des coups et, plus particulièrement, avec le bras gauche lorsqu'un estoc adverse vient du haut de ce même côté. Les livres de combat ne décrivent pas précisément le premier moment de l'action qui consiste à saisir le poignet adverse, avec la main retournée en semi-pronation, le pouce vers le bas. Les textes restent assez généraux; il s'agit plutôt de recevoir l'estoc sur le bras gauche ${ }^{50}$ ou de s'en dé- 
fendre, de le parer ${ }^{51}$. Cette interception doit cependant conduire à la saisie du poignet adverse, afin de lui tordre le bras et attaquer à son tour ou par exemple entreprendre un crochetage aux jambes ${ }^{2}$ (Figure 2).

Or, la mise en pratique montre qu'il est très difficile de venir intercepter les coups de l'adversaire avec une main ouverte et inclinée sans risquer de se retourner le pouce. L'expérimentation conduit à l'hypothèse d'un mouvement semi rotatif partant de la poitrine vers l'extérieur, avec la main ouverte et les doigts serrés, et de venir ainsi intercepter le coup adverse entre le métacarpe et l'avant-bras, puis de refermer la main53. La rotation du poignet

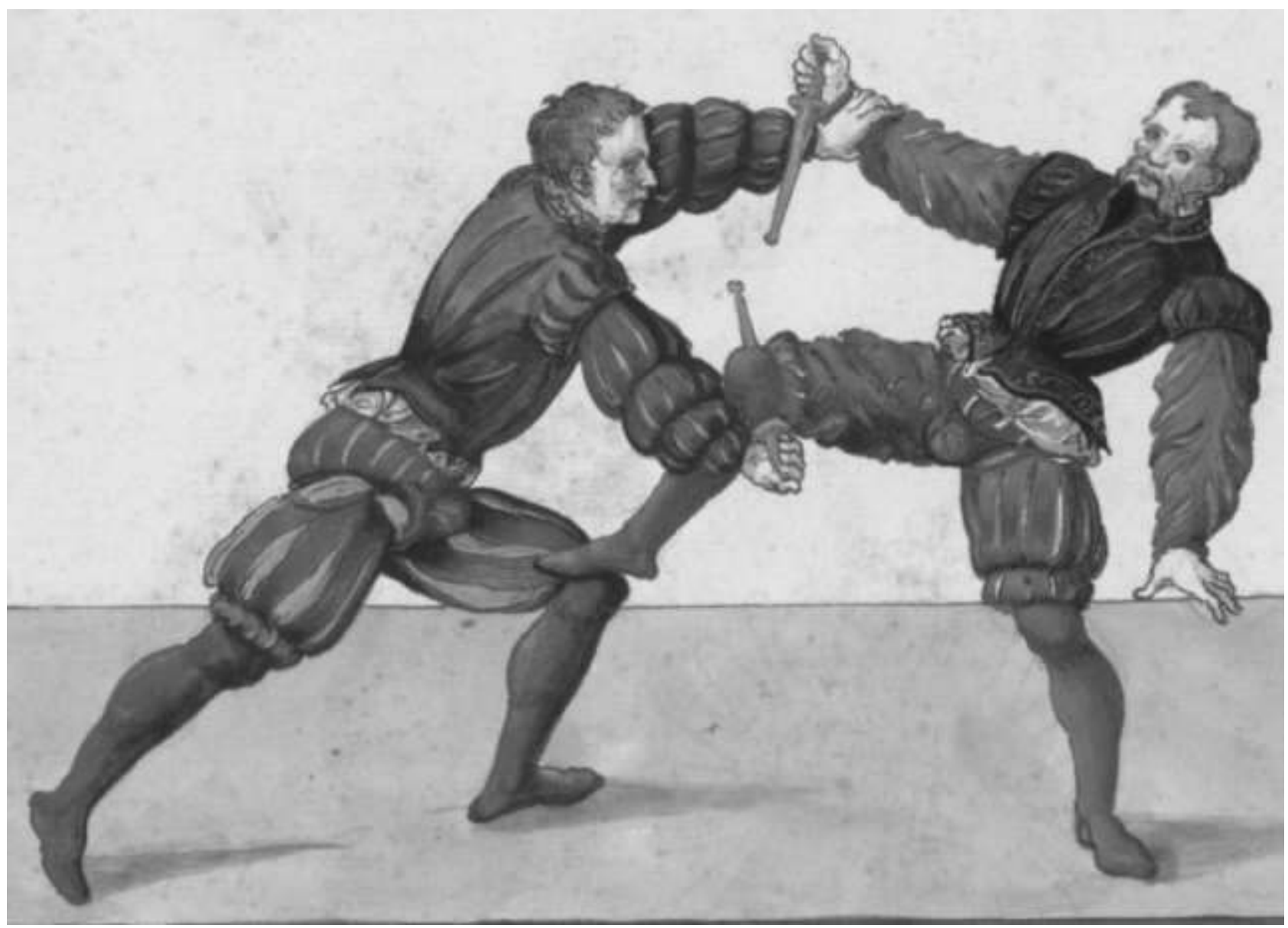

Figure 2 : P.-H. MAIR, milieu du XVIe siècle, Dresde, Sachsische Landesbibliothek, ms. Dresd. C.94, pl.16, $f^{\circ} 10^{\circ}$. 
adverse conduit à l'extension du bras et à son immobilisation. La feinte (feler) est également mal exposée dans les livres de combat, par exemple pour ce qui concerne les tactiques de deuxième intention. Celles-ci consistent « à l'aide d'une feinte, d'une invite, ou d'une fausse-attaque, à suggérer à l'adversaire, l'exécution d'un geste, à provoquer chez lui une réaction qu'on exploitera, et qui permettra de développer une action finale 54 ». C'est le cas par exemple pour une technique illustrée au XVe siècle et reprise au XVIe siècle par Paul-Hector Mair, où, en faisant semblant de frapper une cible haute, on exécute un demi-tour sur les talons et un estoc au pubis55. Ce genre de feinte illustre la difficulté pour un individu non entraîné de se défendre efficacement 56 . Outre la relation d'opposition entre l'enchaînement technique suivant: 1. la pièce (stucke) et le moyen de le contrer : 2. la brisure (brechen), l'expérimentation gestuelle met également en évidence l'existence d'une hiérarchisation des estocs (stechen) en fonction de leur hauteur et de leur orientation 57 . Cette classification comparée à la manière dont le défenseur tient son arme forme un véritable système comprenant toutes les situations et proposant un éventail de possibilités. C'est ce qu'illustre ces tableaux (Tableaux 1 et 2) constitués à partir de cent-vingt-six pièces et de leurs contre, toutes issues des ouvrages de Paul-Hector Mair58.

\begin{tabular}{|c|c|c|c|c|}
\hline $\begin{array}{c}\text { Type d'estoc } \rightarrow / \\
\text { situation du } \\
\text { défenseur } \downarrow\end{array}$ & $\begin{array}{c}\text { Oberstich } \\
\text { (estoc du haut) } \\
\text { visant la tête ou } \\
\text { la poitrine }\end{array}$ & $\begin{array}{c}\text { Oberstich visant } \\
\text { le cou ou la gorge }\end{array}$ & $\begin{array}{c}\text { Oberstich } \\
\text { visant le côté } \\
\text { gauche }\end{array}$ & $\begin{array}{c}\text { Oberstich } \\
\text { visant le côté } \\
\text { droit }\end{array}$ \\
\hline $\begin{array}{c}\text { Dague en tenue } \\
\text { pic à glace }\end{array}$ & 12 & - & 3 & 1 \\
\hline $\begin{array}{c}\text { Dague en tenue } \\
\text { épée }\end{array}$ & 3 & - & 1 & 14 \\
\hline Mains nues & 33 & 6 & - & 1 \\
\hline
\end{tabular}

Tableau 1 : Pièces et brisures des estocs supérieurs. 


\begin{tabular}{|c|c|c|c|c|}
\hline $\begin{array}{c}\text { Type d'estoc } \rightarrow / \\
\text { situation du } \\
\text { défenseur } \downarrow\end{array}$ & $\begin{array}{c}\text { Zwerchstich } \\
\text { (estoc transveral) } \\
\text { au cou ou bien à } \\
\text { la tête }\end{array}$ & $\begin{array}{c}\text { Mitelstich } \\
\text { (estoc du milieu) }\end{array}$ & $\begin{array}{c}\text { Mitelstich } \\
\text { visant la } \\
\text { poitrine en } \\
\text { tenue épée }\end{array}$ & $\begin{array}{c}\text { Unterstich } \\
\text { (estoc du bas) } \\
\text { visant le côté } \\
\text { ou le pubis }\end{array}$ \\
\hline $\begin{array}{c}\text { Dague en tenue } \\
\text { pic à glace }\end{array}$ & 2 & - & - & 2 \\
\hline $\begin{array}{c}\text { Dague en tenue } \\
\text { épée }\end{array}$ & - & - & 1 & 5 \\
\hline Mains nues & 8 & 5 & - & 33 \\
\hline
\end{tabular}

Tableau 2: Pièces et brisures des estocs transversaux, médians et inférieurs.

\section{De l'expérimentation aux lettres de rémission}

On pourrait penser que la majorité des techniques issues des livres de combat fonctionne davantage contre des individus francs et déterminés, lesquels profitent de leur initiative pour exécuter leur attaque, sans se douter qu'une action défensive peut leur être efficacement opposée. Mais cette interprétation est sans doute excessivement influencée par notre vision actuelle du combat au couteau, issue des différents arts martiaux ou de la pratique du self defense. Inversement, les enregistrements vidéo de plusieurs agressions contemporaines et les propos de personnes ayant eu l'expérience de ce genre de situation 59 montrent que certains enseignements actuels sont éloignés de la réalité. Lors d'une agression, l'individu qui n'a pas d'arme est incapable de ménager l'ardeur de son adversaire. Celui-ci peut alors facilement réitérer ses coups une dizaine de fois, et le changement naturel d'orientation des estocs et des angles d'attaque suffisent à ne laisser aucune chance à la victime. Cette observation, que nous avons aussi faite durant nos expérimentations, manque de pertinence. D'une part, elle ne prend pas en compte les paramètres liés à la codification d'un art martial historique. D'autre part, il s'agit finalement de comparer un système technique ancien à une pratique notionnelle contemporaine, au risque de souligner seulement l'aspect théorique, voire peu convaincant, des livres de combat comme des arts martiaux en général. Pourtant les lettres de rémission témoignent de l'importance d'autres facteurs. Par exemple, le fait que les deux protagonistes soient armés change significativement la nature de l'affrontement : on passe alors du statut d'agression à celui de combat, où il n'y a plus réellement d'effet de surprise et où le dénouement de l'affrontement est inconnu des deux adversaires. On remarque également que les coups donnés sont très rarement multiples, ce qui peut supputer l'absence d'acharnement ou, dans certains cas, de la volonté de tuer6o. Étonnamment, le fait qu'il n'y ait pas de règles préétablies déterminant la fin du combat et que les deux individus soient armés, permet en quelque sorte de limiter la violence de l'affrontement et la mort immédiate. Dans ce cas, les techniques de combat semblent pouvoir 
s'appliquer efficacement, d'autant que les coups sont donnés avec une certaine franchise, visant à mettre hors de combat son adversaire avec un seul coup, sans risquer seulement de le blesser superficiellement. Ce qui entraîne souvent dans les lettres la poursuite du combat et la montée de la tension, et le risque pour l'offenseur d'être blessé à son tour. Notons par ailleurs que les individus déterminés sont très présents dans les lettres de rémission et que cette motivation est souvent due à la peur, à la colère ou à l'alcool, « passions » qui conduisent à un certain sentiment de confiance, voire à de l'inconscience. Certains individus ne prennent alors plus en compte la dangerosité de l'arme qui leur fait face et même des coups qui leur sont alloués :

«[...] Ledit Raniequin qui incontinent en lui injuriant de parolles tira un grant braquemart et en frappa ledit suppliant d'estocq et lui en donna deux playes en sa cuisse senestre et fist sa puissance de l'occir et tuer. Pour a quoy obvier ledit suppliant saisi et print en ses bras icellui Raniequin et lui dit qu'il laissast aller sondit bracquemart ou il lui feroit vilonné, ce que ledit suppl Raniequin ne volt fere ains s'efforca de tirer ung dollaquin ou autre petit couteau qu'il avoit pour en occir ledit suppliant, lequel pour a ce remedier et pour contraindre ledit Raniequin a laissier aller ledit braquemart, tira une petite daguette qu'il avoit en sa chainture et lui en donna deux cops vers ses fesses, dont ledit Raniequin ne tint guerres grant compte ains de plus en plus s'efforcoit de vouloir occir ledit suppliant. Lequel considerant que s'il habandonnoit ledit Raniequin et le laissoit aller garny dudit braquemart qu'il s'en repentiroit. Il lui dit que s'il ne laissoit aller icellui braquemart qu'il l'occiroit, de quoy icellui suppl-Raniequin ne tint oncques compte Mais en continuant tousiours ses rudesses s'efforcoit de plus en plus de lui oster la vie. Pour a quoy obvier icellui suppliant frappa ung cop ledit Raniequin de sadicte daguette en la poitrine, duquel cop icellui Raniequin trouva brief apres vie par mort. " ${ }^{61}$

Au demeurant, cette situation n'est pas très éloignée d'un assaut ludique où les coups ne sont pas toujours pris en compte, consciencieusement ou non, par l'un des deux partis, ce qui ne renvoie pas seulement aux livres de combat, mais aussi plus généralement à l'histoire de l'escrime et singulière- ment au problème des tactiques fondées sur les réactions de l'adversaire et l'usage de simulateurs (armes non aiguisées et dépourvues de pointes). Ces problèmes que nous subissons lors de certains assauts libres avec nos dagues en bois, rappellent les propos d'un maître d'armes écossais du XVIIe siècle, William Machrie, pour l'usage du fleuret :

« La raison, dit-il, pour laquelle un artiste peut recevoir une botte d'un ignorant est celle-ci : dans un assaut, on tire généralement avec des épées émoussées, et, quand un ignorant qui estime trop peu l'art de l'épée et se fie à sa seule hardiesse, est prié par un artiste de montrer son jeu naturel, il sait très bien qu'on ne peut lui faire grand mal avec un fleuret émoussé. Aussi s'élance-t-il en avant, quel que soit le nombre de bottes qu'il reçoit, jusqu'à ce qu'il ait touché l'artiste par un coup de hasard ou qu'il arrive si près de celui-ci, qu'il oblige à s'escrimer corps à corps avec lui. Si cet ignorant a seulement touché l'artiste une seule fois, bien qu'il ait reçu trois ou quatre bottes depuis le commencement, il pense avoir remporté la victoire et prouvé que tout n'est que vanité dans l'art de l'escrime. Tandis que s'ils jouaient avec de véritables armes, bien affilées, ou avec des fleurets, ayant seulement une pointe d'un quart de pouce audelà du bouton, je doute qu'il soit difficile de refroidir sa vigueur et son élan. L'ignorant accorderait alors plus d'attention à ce qu'il fait, car il est naturel, même aux hommes les plus hardis et les plus courageux, de tâcher de se garantir, en arrêtant un peu leur poursuite quand ils voient en face d'eux une pointe aiguë prête à les blesser, et qu'ils savent qu'ils courent le risque, sinon de perdre la vie, au moins d'être punis de leur témérité par une blessure. Voilà les raisons pour lesquelles un artiste peut recevoir une botte en coup fourré d'un ignorant. " ${ }^{62}$

Ces considérations sur le combat au poignard tendraient à l'isoler des autres armes plus conventionnels et à voir en son art une pratique davantage adaptée à la défense personnelle qu'à celle de l'escrime. Ceci est sans compter certaines « adaptations » que peut subir le combat au poignard. Quand deux adversaires compétents sont face à face, deux «maitres » comme les textes les appellent, les techniques fondées sur l'ignorance ou la surprise de l'assaillant n'ont plus la même pertinence: les feintes, les ruses, les changements de 
rythme font qu'agir " après 63 » son adversaire est souvent fatal. A l'opposé, agir avec trop de précipitation l'est également. Certes, il semble difficile d'exécuter correctement une technique sur une attaque réalisée avec force et vitesse, mais nos expériences montrent les possibilités qu'offre une telle connaissance technique. Premièrement, il s'agit de savoir intercepter intelligemment le coup porté. Deuxièmement, de commencer une technique afin de faire prendre conscience à l'adversaire les risques encourus par sa démarche. Troisièmement, si l'adversaire arrive à s'en défendre, de changer de technique ou de l'estoquer afin de reprendre l'initiative.

Ainsi, les techniques durant nos assauts sont rarement exécutées entièrement, mais leur existence conduit à une certaine profondeur tactique. PaulHector Mair expose souvent ces possibilités d'enchaînements, comprenant plusieurs actions de la part des deux adversaires. Il montre aussi dans ses premières pièces des situations où l'on vient réciproquement à l'engagement de la lame ou du poignet adverse après une éventuelle interception ${ }^{64}$. Ces actions sont suivies comme les autres par une suite d'attaques, de parades et de contre-attaques. Il s'agit bien alors d'une pratique martiale, davantage fondée sur la sureté du jugement et de l'engagement 65 et non plus une escrime intuitive dépendante des trop nombreux paramètres que nous avons rencontrés.

\section{Conclusion}

Nous avons vu, à la comparaison des différentes sources, que l'emploi d'un couteau, d'une dague ou d'un poignard est universel, puisqu'il suit le profil technologique de ce type d'arme, à savoir l'utilisation de la pointe pour donner des coups d'estoc. Cependant on constate, à la lecture des livres de combat, qu'une utilisation technique est possible, mais absente des lettres de rémission. Outre les spécificités rédactionnelles liées à ce type de document, où le suppliant ne peut ni mettre en avant sa prestation technique, ni se souvenir de l'ensemble des détails, on notera différentes raisons de cette absence, a priori, d'élément techniques : premièrement, les situations réelles sont improvisées et les différents facteurs, physiques comme psychologiques, conduisent à la simplification de l'affrontement. Deuxièmement, l'optimisation du geste offensif n'est pas nécessairement recherchée, car l'objectif est plus souvent de mettre fin à une situation que de tuer. Troisièmement, le combat technique à la dague fait appel à une participation active de l'ensemble du corps (comme l'utilisation du bras gauche), principe qui n'est pas inné. Ainsi le système technique conçu par les maîtres d'armes à partir des coups de base et de la tenue de l'arme semble d'autant plus pertinent, aussi bien par son aspect offensif, en trompant la défense adverse, que par son aspect défensif, basé sur l'ingénuité des attaques de l'adversaire. Cependant, comme le contexte et la nature de l'armement utilisé déterminent certaines possibilités, d'autres paramètres sont à prendre en compte dans la pratique sérieuse ou ludique, comme la détermination ou l'ignorance, facteurs à double tranchant pour le maître d'armes voulant appliquer sa noble science. Ainsi dans certains cas, celui-ci est amené à changer sa pratique et à se diriger vers une escrime plus conventionnelle, celle de l'engagement, lui permettant de prendre moins de risque. Ainsi, les livres de combat sont fondés sur une pratique à la frontière entre la réalité du combat à la dague et les paramètres théoriques, liés à la codification d'un art martial. En définitive, la pratique expérimentale a permis à la fois d'analyser les différentes techniques des livres de combat et d'entreprendre une nouvelle lecture des sources judiciaires. Elle n'a pas été une finalité, mais le moyen de relier deux sources que tout opposait: les livres de combats et les lettres de rémission, mais aussi la théorie et la pratique pour poignarder un homme... 
1 Cf. L'épée, usages, mythes et symboles, catalogue d'exposition, Musée national du Moyen Age de Cluny, Paris, 2011.

2 Par exemple l'ire (la colère) dans les sept péchés capitaux, est représentée par un sanglier chevauché par un homme en train de poignarder un individu, dans BELLOVACENSIS Vincentius, Speculum historiale (trad. Jean de Vignay), 1453, Paris, BNF, Français 50, fol.25r ${ }^{\circ}$.

3 Ex: BOCCACCIO Giovanni, Libération d'Arcitas, 1470, Vienne, Österreichische Nationalbibliothek, ms. 2617, fol.64r ${ }^{\circ}$.

4 Comme les dagues dites « à couillettes » ou à « rognons ».

5 «Daghes ou cuteals de teile longeche, grosseche et largheche que ensengniet sierat ", dans l'ordonnance de Jean de Bavière pour la répression des crimes et des délits dans la cité de Liège, datant du 24 février 1395, dans RAIKEM J.J., POLAIN M.L. et BORMANS S., Coutumes du pays de Liège, Bruxelles, t.2, 1873, p. 84-86. "On défend de porter tous couteaux à pointe démesurée, sous peine d'une amende de trois livres et de confiscation des couteaux; celui qui les porterait autrement qu'à sa ceinture, forfairait cent escalins et le couteau à moins que la pointe n'en fut tellement brisée que les échevins jugeraient que ces armes sont inoffensives », dans LIMBURGSTIRUM Th. de, Coutumes des pays et comté de Flandre, quartier de Gand, coutumes de la ville d'Audenarde, Bruxelles, t.4, 1882, p.122.

6 C'est ce que montre l'étude des documents normatifs, lesquels légalisent rarement le port ou l'utilisation d'une arme. Ces faits sont confirmés aussi par les lettres de rémission, par lesquelles un justiciable obtient l'absolution de son crime. $C f$. BAS PierreHenry, "Pour la deffense et tuicion de leur corps », théories et pratiques martiales dans les sociétés franco-bourguignonne et germanique, de la fin du Moyen Âge au milieu de la Renaissance. Thèse de doctorat en cours sous la direction de Bertrand Schnerb.

7 Der degen / ader das kurcze messer : «l'enseignement de la dague ou bien le court couteau » commence assez timidement avec le premier manuscrit connu de la tradition du maitre d'armes Johannes Liechtenauer, le ms. 3227a attribué à tord à DÖBRINGER Hanko, circa 1389 ( ?), Nuremberg, Bibliothek des Germanischen Nationalmuseums, fol.84 $\mathrm{r}^{\circ}-85 \mathrm{r}^{\circ}$. Il sera encore présent dans l'ouvrage de MEYER Joachim, Gründtliche Beschreibung des Fechtens, Strasbourg, 1570.

8 Ordonnance des échevins de Termonde, datant du XVe siècle, dans LIMBURG-STIRUM Th. de, Coutumes des pays et comté de Flandre, quartier de Gand, coutume de la ville de Termonde, t.6, 1896, p.312-320.
9 WARD PERKINS John Brian, London Museum, Medieval Catalogue, Londres, 1967, p.53.

10 Par ex. Archives Départementales du Nord (Noté dorénavant ADNord) (B 1681, fol.66r ${ }^{\circ}$ ), Arras, novembre 1389.

11 Ce terme semble avant tout être un synonyme pour désigner une dague, $c f$. la grande charte des Gantois datée du 8 avril 1297 (n.s.), GHELDOLF A. E., Coutumes des pays et comté de Flandre, Coutume de la ville de Gand, t.1, Bruxelles, 1868, p.444 et Misericoerden, cf. Ordonnance des échevins de Termonde, op.cit. (note 7).datant du XVe siècle, LIMBURG-STIRUM, 1896, p.312-320. Grande charte des Gantois, 8 avril 1297 (n.s.).

$12 \mathrm{Ou}$ petit cousteau espointé, AN (JJ 217, fol.76 $6^{\circ}$, Ancenis, juillet 1487. Lettre citée par Vanessa MORINEAU, Le crime pardonné d'après les lettres de rémission en 1487, Mémoire de Master sous la direction de Michel Nassiet, Université d'Angers, 2009. http://www.sites.univrennes2.fr/cerhio/IMG/pdf/Lettres_remission_1487.pdf (consulté le 15 mars 2012)

13 ADNord, (B 1698, fol.3v $v^{\circ}-6 r^{\circ}$ ), Malines, juin 1475, pour une première mention assez tardive pour le XVe siècle et ADNord (B 1726 , fol. $73 \mathrm{r}^{\circ}-73^{\circ}$ ), Bruxelles, octobre 1514 , pour l'une des plus tardives. On trouve les deux termes ensemble dans certains textes normatifs : "Qui est trouvé portant baston defendu si comme lance de fer, ou de plomb, de hache, couteau a pointe ou dollequin, chet en amende de soixante sols ", BOUTILLIER, Somme rurale, 2e p., fol.64b, éd.1486, cité par GODEFROY Frédéric, Dictionnaires de l'ancienne langue française et de tous les dialectes du IXe au XVe siècle, 10 vol., New York, 1961 (reprint de l'édition de Paris, 1881-1902), t.2, p.728.

14 Terme lié aux origines latines : pugio, onis, $\mathrm{m}$ : le poignard (des gladiateurs) et pugno, as, are, avi, atum : combattre à coup de poing, GAFFIOT Félix, Dictionnaire latin-français : Le grand Gaffiot, Paris, Hachette, 2002.

15 Á noter qu'en flamand au XVIIe siècle il semble exister encore une différence entre le poignard (poingnaerden) et le couteau à pointe (opsteeckers), $c f$. la landcharter des pays et baronnie de Grimberghen de novembre 1606, Constant Casier, Coutumes du pays et duché de Brabant, t.2, Bruxelles, 1873, p.238-239.

16 Tolchen odder Kaempfftegen : "poignard ou bien dague de combat", anonyme, Der Altenn Fechter anfengliche Kunst, Francfort-sur-le-Main, 1531, p.31.

17 Paul-Hector Mair (1517-1579), homme politique d'Augsbourg.

18 Mais aussi Dolch ou Tolch, cf. MAIR Paul-Hector, milieu du XVIe siècle, Dresde, Sachsische Landesbibliothek,. Ms. Dresd. C.93 et C.94. En latin : Pugionis, Id., De Arte Athletica, milieu du 
XVIe siècle, Munich, Bayerische Staatsbibliothek, Cod.icon.393, t. I et II.

19 Est-ce que les deux noms ont pour origine commune le latin dolo, dolon, onis, $\mathrm{m}$ : une arme courte ou un poignard chez les romains, ou est-ce que dolchen serait d'origine slave ? cf. J. et W. GRIMM. Deutsches Wörterbuch, Leipzig, t.2, 1854-1961, Sp.1222. Quellenverzeichnis, Leipzig, 1971. Merci à Jean.Dominique Delluche pour cette dernière précision.

20 ADNord, (B 1689, fol.19v ${ }^{\circ}-20 r^{\circ}$ ), Bruxelles, juin 1459.

21 Ici le grand ou long couteau, appelé également braquemart ou messer dans les livres de combat, est un couteau pouvant avoir la même taille qu'une épée.

22 ADNord, (B 1704, fol.1r ${ }^{\circ}-2 v^{\circ}$ ), Gand, août 1484.

$23 C f$. la majorité des livres de combat présentant le duel en armure.

24 AN, (JJ 246, fol.39r ${ }^{\circ}$ ), Rouen, février 1532. Lettre de rémission éditée par Michel Nassiet, http://www.sites.univrennes2.fr/cerhio/IMG/pdf/Letrres_remission_1531-1532.pdf

25 Hatt vil vortails. so zu der erhalltung des lebens diennet : MAIR Paul-Hector, ms. Dresd. C93, op. cit., fol.17v ${ }^{\circ}$.

26 Exemple de " la septième règle » d'Andreas Lignitzer, Item hat er sein tolchen. Ehe gezogen dann du den deinen. vnnd stich dir oben : "De même s'il a tiré son poignard plus tôt que toi le tien et qu'il t'estoque du haut ", reprise dans les ouvrages de MAIR Paul-Hector, ms. Dresd. C94, op. cit., fol.28v .

27 Aüsz dem Dolchen kumbt das Ringen. vnnd werden vast. mit vnd durch ainannder geregiert vnd gelernet : " À partir du poignard vient la lutte / et elles seront parfaitement maitrisées et apprises l'une avec et à travers l'autre», Id., ms. Dresd.C93, op. cit., fol. $17 \mathrm{v}^{\circ}$.

$28 C f$. illustration 1 et MAIR Paul-Hector, ms. Dresd. C94, op. cit., fol. $3 \mathrm{r}^{\circ}$ pl.1-fol. $24 \mathrm{v}^{\circ}$ et $I d$., milieu du XVIe siècle, Vienne, Österreichische Nationalbibliothek, cod. Vind. 10825, fol.19or ${ }^{\circ}-$ $226 \mathrm{r}^{\circ}$.

29 Notamment les poignets, les fosses cubitales, les creux poplités et le cou.

30 notera justement que dans le De Arte Athletica, op. cit., les dagues ne sont plus en bois, mais en métal, ainsi certaines actions consistant à prendre la lame de l'adversaire sont exécutées par des individus équipés de gants de cuir. $C f$. pl.24, fol.14v $\mathrm{v}^{\circ}$ et pl.36, fol.20v ${ }^{\circ}$.

31 nimbt er sein tolchen Inn die hannd also. das die klingen vnnden an der hand aufgeet. so will er von oben zu dir stechen :
«S'il prend ainsi son poignard dans la main de sorte que la lame se lève par-dessous la main : alors il veut t'estoquer depuis le haut ", dans MAIR Paul-Hector, ms. Dresd. C94, op. cit., fol. $27 \mathrm{r}^{\circ}$.

32 Nimpt er aber den tolchen Inn die hannd also. Das die klingen oben bey dem arm aufgeet. so will er von vnnden auf dich stechen : "Mais s'il prend le poignard dans la main de sorte que la lame se lève près du bras, alors il veut t'estoquer du bas vers le haut », Id., Ibid. Ou encore : "Sil a son poingnart et petit doiz enuers lalumelle, dans anonyme, La Noble Science des ioueurs despée, Anvers, 1538, p.35.

33 "Il la frappa du plat de sa dague sur les espaulles, sans lui faire mal », AN, (JJ 217, fol.111v ${ }^{\circ}-113 \mathrm{r}^{\circ}$ ), Rouen, Novembre 1487. Lettre citée par MORINEAU Vanessa, Le crime pardonné..., 2009, op. cit.

$34 \mathrm{Cf}$. illustration 1

35 Par ex.: "Combien qu'il n'eust pas propoz, ne vouloit le frapper a mort ledit Tachet, lessa sa dicte dague qu'il tenoit nue, et d'icelle frappa ledit Tachet sur le bras senestre, tellement qu'il le lui persa tout oultre et en glissant passa et roula la dicte dague jusque a la mammelle dudit Tachet, tirant au cuer tellement que dudit cop icelui Tachet cheut par terre et depuis ne parla, puis assez tost après termina vie par mort ", ADNord, (B.1692, fol.19v $\left.{ }^{\circ}-21 r^{\circ}\right)$, Lille, janvier 1467.

36 Par ex. : entre le col et l'espaulle, ADNord, (B.1700, fol.91r ${ }^{\circ}$ $\left.91 v^{\circ}\right)$, Bruxelles, décembre, 1479.

37 Par ex. : «le cuidant seulement batre et non tuer lui donna ung seul cop [d'une vouge] en la cuisse duquel cop tantost après il termina vie par mort », ADNord, (B.1703, fol.53v $\left.\mathrm{v}^{\circ}-54 \mathrm{v}^{\circ}\right)$, Bruges, février, 1482.

38 Dann es ist zu merckhen das alweg der Elnpogen der hannd mit dem stosz nachvolgen soll. dann es gibt Im ain gwalt vnnd guoten nach truckh : "Ensuite, ceci est à retenir, que le coude doit toujours suivre la main avec la percussion. Alors il lui vient une violente et bonne poussée ", dans P.-H. MAIR, ms. Dresd. C94, op. cit., fol.27r ${ }^{\circ}$.

39 « Expériences » menées dans un cadre contemporain et ludique, au sein de l'association nordiste REGHT (Recherche et Expérimentation du Geste Historique et Technique).

40 Comme ceux utilisés pour le combat réel au couteau, PENTECOST Don, Put'em down, take 'em out! Knife Fighting Techniques from Folsom Prison, Boulder, Paladin Press, 1998.

41 Si le Ms.3227a, circa. 1389 ?, op.cit. (note 6) mentionne ce genre de manifestation, les sources concernent davantage une période bien plus tardive, cf. WASSMANNSDORFF Karl, Sechs 
Fechtschulen der Marxbrüder und Federfechter aus den Jahren 1573 - 1614, Heidelberg, 1870.

42 Notamment Martin HUNTZFELTZ et LIGNNITZERS Andres dans DANTZIG Peter, 1452, Rome, Biblioteca dell'Accademia Nazionale dei Lincei e Corsiniana, Cod. 44 A 8 (Cod. 1449), fol. $85 v^{\circ}-86 r^{\circ}$ et $94 r^{\circ}-96 r^{\circ}$. Ou encore TALHOFFER Hans, 1467 , Munich, Bayerische Staatsbibliothek, Cod. Icon. 394a , pl. 170190.

43 Es ist furnemlich acht zuhaben alle stich der massen $\mathrm{Zu}$ erfaren / das sie nit angeen vnnd damit sich aber ainer am ersten befleisz dem gogen man mög abpruch thun vnnd beschliessen : Ilest essentiel de veiller à avoir appris tous les estocs de telle sorte qu'ils ne puissent pas attaquer (atteindre), mais qu'avec ceci, si quelqu'un s'empresse le premier contre, que l'on puisse lui faire une brisure de bras et un verrouillage, MAIR Paul-Hector, ms. Dresd. C94, op. cit., fol.27r ${ }^{\circ}$.

44 "Der stich fry », un estoc du haut donné avec la jambe gauche laissée devant (?), TALHOFFER Hans, 1467, op. cit., pl.171.

45 ADNord (B.1722, fol.11r ${ }^{\circ}-12 \mathrm{r}^{\circ}$ ), Gand, mai 1511 et MAIR PaulHector, ms. Dresd. C94, op. cit., fol. $45 \mathrm{v}^{\circ}$ et $46 \mathrm{r}^{\circ}$.

46 ADNord (B.1713, fol.40v ${ }^{\circ}-42 r^{\circ}$ ), Malines, juillet 1502.

47 AN (JJ 169, fol.21v ${ }^{\circ}-22 r^{\circ}$ ), Paris, janvier 1416.

48 AN, (JJ 246, fol.13vº), Fontainebleau, août 1532. Lettre de rémission éditée par Michel Nassiet, op. cit., 2009.

49 ADNord (B.1699, fol.34r $\left.\mathrm{r}^{\circ}-35^{\circ}\right)$, Bruges, septembre 1477 et (B.1712, fol.139v $\left.{ }^{\circ}-140 v^{\circ}\right)$, Malines, novembre 1501 .

50 Par ex. Die sibent Regel : "la septième règle», reprise dans MAIR Paul-Hector, ms, Dresd. C94, op. cit. fol.28v

51 Versetzen, Id., Ibid., fol.29r ${ }^{\circ}$.

52 TALHOFFER Hans, 1467, op. cit., pl.190 et l'illustration $\mathrm{n}^{\circ} 2$.

53 À noter que certaines parties du carpe (principalement l'os pointu appelé pisiforme) sont assez fragiles et que la pointe formée par le styloïde cubitale, à l'extrémité de l'ulna (cubitus) peut être douloureuse pour l'adversaire.

54 CLERY Raoul, L 'escrime (fleuret, épée, sabre), Paris, Amphora, 1965, p.393.

55 Anonyme, appelé "codex Wallerstein », circa 1470, Augsbourg, Universitätsbibliothek, Cod. I.6. $4^{\circ} .2$, fol.26r ${ }^{\circ}$ et MAIR Paul-Hector, ms Dresd. C94, op. cit., fol.10r ${ }^{\circ}$, pl.15. Cf. illustration $\mathrm{n}^{\circ} 1$.
56 Des exercices de ce genre ont été menés à plusieurs reprises par l'association REGHT. Il est vraisemblablement impossible qu'un individu sans expérience, puisse se défendre correctement face à un individu expérimenté dans le maniement de la dague.

57 Oberstich: «estoc du haut» partant de l'épaule droite; zwerstich: " estoc transversal» partant de l'épaule gauche; mitelstich: " estoc du milieu » partant de la hanche gauche; unterstich : « estoc du bas » partant de la hanche droite.

58 Plus précisément le "livre» (das buch) qui concerne la dague, in MAIR Paul-Hector, ms Dresd. C94, op. cit., $\mathrm{f}^{\mathrm{o}} 25 \mathrm{r}^{\circ}-$ $55^{\circ}$.

59 PENTECOST Don, 1998, op. cit.

60 Bien évidement pour sa défense, le suppliant justifiera très souvent son geste par le souhait de mettre simplement fin au conflit.

61 ADNord (B.1700, fol.87r ${ }^{\circ}-88 r^{\circ}$ ), Amiens, Mars 1479.

62 MACHRIE William, The Sword-Man's Vade mecum : or a preservative against the surprize of a sudden attaque with sharps, Edimbourg, 1691, p.58-59. Cité dans CASTEL Egerton, L'escrime et les escrimeurs depuis le Moyen Age jusqu'au XVIIIe s., esquisse du développement et de la bibliographie de l'art de l'escrime pendant cette période, illustré de reproductions de vieilles estampes et de photogravures, traduction A. Fierlants, Paris, 1888. p.224-225.

63 Le nach, "l'après » s'oppose dans les textes germaniques au vor, « l'avant », c'est-à-dire pour simplifier, à la prise d'initiative.

64 Par exemple, MAIR Paul-Hector, ms Dresd. C94, op. cit., fol. $3 \mathrm{r}^{\mathrm{o}}-4 \mathrm{v}^{\circ}$, pl.1-4.

65 L'anpund ou contactus, us, $\mathrm{m}$ : le liement, le contact. 